Original Research Article

\title{
A pharmaco-epidemiological study of psoriasis
}

\author{
Swikriti Gupta $^{1}$, Swathi Acharya ${ }^{2 *}$, Girisha Banavasi Shanmukha ${ }^{3}$, Rajendra Holla ${ }^{2}$
}

\begin{abstract}
${ }^{1}$ MBBS, ${ }^{2}$ Department of Pharmacolgy, ${ }^{3}$ Department of Dermatology, K.S. Hegde Medical Academy, Deralakatte, Nitte Deemed to be University, Mangalore, Karnataka, India
\end{abstract}

Received: 30 August 2019

Revised: 07 October 2019

Accepted: 09 October 2019

\section{*Correspondence to:}

Dr. Swathi Acharya,

Email: acharyaswathi4@ gmail.com

Copyright: (C) the author(s), publisher and licensee Medip Academy. This is an openaccess article distributed under the terms of the Creative Commons Attribution NonCommercial License, which permits unrestricted noncommercial use, distribution, and reproduction in any medium, provided the original work is properly cited.

\begin{abstract}
Background: Psoriasis is a chronic relapsing disease necessitates long-term therapy. The disease severity, comorbidities, and access to health care determine the choice of therapy for psoriasis. The main aim of the treatment of psoriasis is to induce prolonged remission and suppress the disease as there is no cure. It is only palliative and symptomatic treatment, and the choice of treatment modality is according to the type of presentation. The various modalities of treatment available include topical therapies, systemic therapies, phototherapy, and biologics. The difference includes among clinical varieties in their presenting features, severity, natural course, and response to the treatment are responsible for variation in choice of therapy.

Methods: In this descriptive study, data collected from patients diagnosed with psoriasis who were admitted in a K.S. Hegde Charitable hospital from January 2017 to January 2019 were analysed for demographic features, disease pattern and drug use pattern in patients.

Results: Psoriasis vulgaris/plaque type of psoriasis was commonly observed type of psoriasis in our patients with fourth and fifth decade patients getting more affected. Diabetes was the most commonly associated comorbid disorder. The patients were treated both with systemic and topical modalities of treatment, antihistaminic and methotrexate were most commonly most commonly used systemic drug and moisturizers and glucocorticoids were used topical drugs.

Conclusions: Psoriasis needs a multimodality treatment with careful monitoring for the comorbid disorder. Treatment choice is individualised depending on the severity and body parts affected.
\end{abstract}

Keywords: Psoriasis, Glucocorticoid, Methotrexate, Psoriasis vulgaris

\section{INTRODUCTION}

Psoriasis, an inflammatory condition of the skin characterized by red, scaly, sharply demarcated, indurated plaques, mainly affecting the extensor surfaces and the scalp. The worldwide burden of psoriasis is $2 \%$, and the prevalence of psoriasis in India ranges from $0.44 \%$ to $2.8 \% .^{1,2}$ It has been increasing due to the modern stressful lifestyle, lack of adequate physical activities, and associated risk of metabolic syndrome. ${ }^{2}$ Depending on the lesion characteristic feature and the parts of the body affected, there are various types of psoriasis. Main five types are Plaque psoriasis, guttate psoriasis, Inverse (Flexural) psoriasis, pustular psoriasis, and erythrodermic psoriasis. ${ }^{3}$ The differences among clinical varieties in their presenting features, severity, natural course, and response to the treatment are responsible for variation in choice of therapy. ${ }^{2}$

Psoriasis has significant morbidity due to lifelong disease course with a high impact on the person's economy and the psychological and social burden, which affects the quality of life of patients. ${ }^{4}$ It may affect patients' life adversely, for example, emotional status, psychological stress, self-esteem, relationships, work, social activities, financial burden, and even physical function, particularly in patients with psoriasis arthritis. ${ }^{2}$

Psoriasis is considered an immune-mediated inflammatory disorder (IMID), alongside other entities such as rheumatoid arthritis, Crohn's disease, and 
multiple sclerosis. ${ }^{5}$ The sustained inflammation that leads to uncontrolled keratinocyte proliferation and dysfunctional differentiation is the hallmark of psoriasis. Development and sustainment of psoriatic inflammation are mainly due to disturbances in the innate and adaptive cutaneous immune responses. ${ }^{6,7}$

Psoriasis also has a genetic component. There is an increased incidence of developing psoriasis in first and second-degree relatives of patients. ${ }^{8,9}$ Broad spectrum of environmental factors in addition to genetic factors, has been documented to modulate features, course, and severity of psoriasis. Knowledge of the environmental triggering factors and their avoidance contributes to the cure or reduction of recurrences/exacerbation. ${ }^{10}$

Another shared feature of immune-mediated inflammatory disorder (IMIDs) is the association with other diseases, in particular, cardiovascular diseases. The more commonly associated co-morbidities in psoriasis patient include psoriatic arthritis (PsA) and Crohn's disease, diabetes mellitus, arterial hypertension, rheumatoid arthritis, obesity, ischemic heart disease, depression, and malignancy. ${ }^{11}$

The main aim of the treatment of psoriasis is to induce prolonged remission and suppress the disease as there is no cure. It is only palliative and symptomatic treatment, and the choice of treatment modality is according to the type of presentation.

The various modalities of treatment available include topical therapies, systemic therapies, phototherapy, and biologics. ${ }^{12}$ Intermittent therapy with frequent alterations in treatment options is usually needed to reduce the toxicity of anti-psoriatic drugs.

The topical medications include emollients, dithranol, coal tar, calcipotriol, retinoids, and glucocorticoids (GC). Systemic therapy includes immunosuppressants such as methotrexate (MTX) and cyclosporine, retinoids like acitretin and etretinate, and biological response modifiers such as etanercept, efalizumab, adalimumab, infliximab, and alefacept. Phototherapy involves ultraviolet B irradiation of the skin and also. Recently laser therapy is also being used for the treatment. ${ }^{13,14}$

However, evaluation of the treatment modalities of this disease in a particular area from time to time is required to provide an insight into the pattern of disease, profile of drug use and available novel therapeutic options. Hence the study is planned to observe the disease pattern and drug use pattern for the treatment of different types in our tertiary care setting.

\section{METHODS}

The present study was retrospective, descriptive study conducted in department of dermatology of a K.S. Hegde charitable hospital, Mangaluru. The data from the
Patients of either gender diagnosed with psoriasis and patients admitted in the hospital from January 2017 to January 2019 was collected for the study. Data from patients diagnosed with psoriasis and prescribed with a minimum of one or more medications were included in the study. Data from patients with incomplete case records were excluded.

The institutional ethics committee approval was taken before starting the study. Data regarding patient's demographics and their clinical history and drug history was obtained from the patient records from IPD maintained in the MRD documented in Performa prepared for the study, and the data was analysed.

\section{RESULTS}

Total of 90 patients case records with diagnosis of psoriasis were included for the study admitted during the study period, among these 75 were males $(83.33 \%)$ and 15 were females $(16.66 \%)$ with the mean age of $48.6 \pm 15.31$ years (Table 1 ). Regarding the distribution of disease type majority of the patients were diagnosed with Psoriasis vulgaris/plaque type psoriasis (82.22\%) (Table 2 ) and they presented with the most common complaint of itchy and scaly lesions (71.11\%) followed by complaint of joint pain in $12.22 \%$. Among the 90 patients studied 31 (34.44\%) patients had lesion distributed in whole body and $28.88 \%$ had scalp lesion and $32 \%$ had lesion in upper limb and lower limb and $23.33 \%$ had lesions on abdomen (Table 3). 23 patients had comorbid disorder around 10 of them had diabetes, 10 of them had hypertension and 3 of them had bronchial asthma.

Table 1: Age distribution of psoriatic patients.

\begin{tabular}{|llll|}
\hline Sl.no & Age (in years) & Number of patients & $\%$ \\
\hline $\mathbf{1}$ & $1-10$ & 3 & 3.33 \\
\hline $\mathbf{2}$ & $10-20$ & 2 & 2.22 \\
\hline $\mathbf{3}$ & $21-30$ & 3 & 3.33 \\
\hline $\mathbf{4}$ & $31-40$ & 11 & 12.22 \\
\hline $\mathbf{5}$ & $41-50$ & 21 & 23.33 \\
\hline $\mathbf{6}$ & $51-60$ & 35 & 38.88 \\
\hline $\mathbf{7}$ & $>60$ & 15 & 16.66 \\
\hline
\end{tabular}

Table 2: The types of psoriasis.

\begin{tabular}{|lll|}
\hline Types & No. of patients & $\%$ \\
\hline Psorisis vulgaris & 74 & 82.22 \\
\hline Palmoplantar psoriasis & 2 & 2.22 \\
\hline Psoriatic arthritis & 4 & 4.44 \\
\hline Acute guttate psoriasis & 2 & 2.22 \\
\hline Pustular psoriasis & 3 & 3.33 \\
\hline Erythrodermic psoriasis & 4 & 4.44 \\
\hline Acrodermatitis continua & 1 & 1.11 \\
\hline Total & 90 & 100 \\
\hline
\end{tabular}


Among the 90 patients studied 68 (75.55\%) patients received combination of systemic and topical therapy and around $18(20 \%)$ patients received only topical therapy and $4(4.44 \%)$ patients received only systemic therapy. Total number of the systemic drugs prescribed was 197 with the average number of drugs prescribed being 2.188 and the number patients with polypharmacy i.e., prescribed more than five drugs were $7(7.77 \%)$. The most common group of drug prescribed was antihistaminic $(35.03 \%)$ followed by multivitamins $(17.76 \%)$, antibiotics $(16.75 \%)$ and psoriatic drugs (12.69\%). The most commonly used antihistaminic drug was levocetrizine $(46.37 \%)$ and most commonly used antibiotic was amoxicillin (57.77\%) (Figure 1). Among the psoriatic drugs, methotrexate was most commonly used drug in around $48 \%$ of the patient it was used in oral route and in $24 \%$ used in injection route. Cyclosporine $(8 \%)$ and methyl-prednisolone (8\%) UV B therapy (4\%), apremilast (4\%) were other, psoriatic drugs used (Table 4).

Table 3: The distribution of lesion in psoriasis.

\begin{tabular}{|c|c|c|}
\hline Distribution of lesions & No of patients & $\%$ \\
\hline Whole body & 31 & 34.44 \\
\hline Scalp & 26 & 28.88 \\
\hline $\begin{array}{l}\text { Both upper and lower } \\
\text { extremities }\end{array}$ & 29 & 32.22 \\
\hline Abdomen & 21 & 23.33 \\
\hline Chest & 4 & 4.44 \\
\hline Face & 2 & 2.22 \\
\hline
\end{tabular}

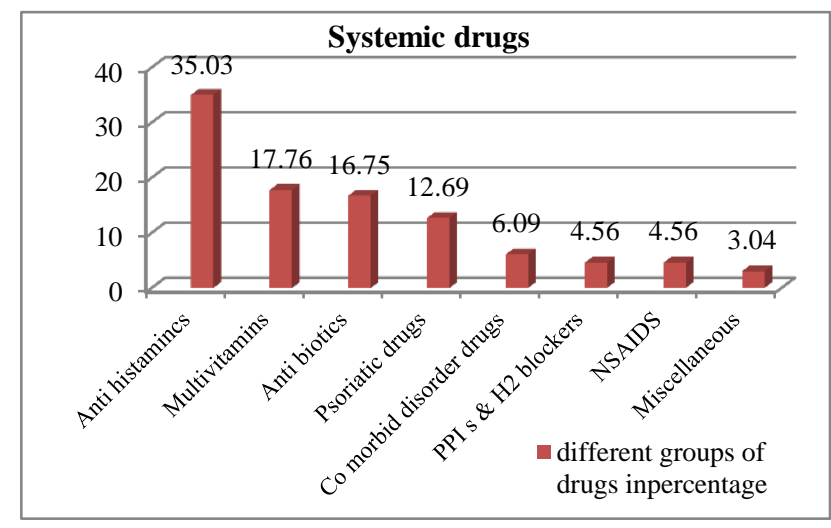

Figure 1: Different groups of systemic drugs used in psoriasis.

Table 4: Different systemic drugs used.

\begin{tabular}{|lll|}
\hline Psoriatic drugs & $\begin{array}{l}\text { Number of } \\
\text { patients }\end{array}$ & $\%$ \\
\hline Tab.Methotrexate & 12 & 48 \\
\hline Inj. Methotrxate & 6 & 24 \\
\hline Cyclosprine & 2 & 8 \\
\hline Methylprednisolone & 1 & 8 \\
\hline Prednisolone & 1 & 4 \\
\hline UVB therapy & 1 & 4 \\
\hline Apremilast & 1 & 4 \\
\hline
\end{tabular}

\section{Topical drugs}

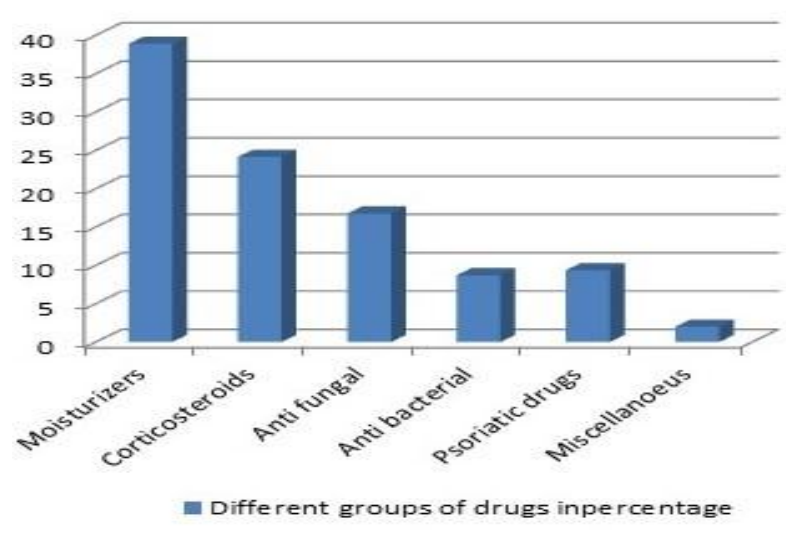

Figure 2: Different groups of topical drugs used in psoriasis.

Total number of the topical drugs prescribed was 149 with the average number of drugs prescribed being 1.65. The most common group of drug prescribed was moisturizers (38.9\%) followed by corticosteroids $(24.16 \%)$, antifungals $(16.77 \%)$ and anti-psoriatic drugs $(9.39 \%)$. The most of the topical preparations were combination of moisturizers and steroid preparation. The most commonly used moisturizer was liquid paraffin $(60.34 \%)$ followed by aleovera moisturizing solution $(18.96 \%)$ and glycerine $(8.62 \%)$. The most commonly used steroid was clobetasole in $46 \%$ of the patients in combination with moisturizer and in $19 \%$ as monotherapy. Other steroid preparations were halbetasole, mometasone, betamethasone and hydrocortisone. The other commonly used topical medications were coal tar $(71 \%)$ in combination with keratolytic agent salicylic acid and calcipotriol (28.57\%) (Figure 2).

\section{DISCUSSION}

Our study aimed at evaluating the disease pattern and drug use pattern in psoriasis patients receiving in a tertiary care hospital. Our study included patients diagnosed with psoriasis and prescribed with a minimum of one or more medications. Among the 90 patients studied, the majority of them were males around $75(83.33 \%)$ indicating the male predominance. This was similar to the findings of study by Pradyumana et al, Karamata et al, Raghunandan et al. ${ }^{11,15,16}$ The mean age of patients in our study was $48.6 \pm 15.31$ years with majority of them belonging to the fourth $(23.33 \%)$ and fifth $(38.88 \%)$ decade of life. This was similar to the findings in study by Dogra et al and Ayanlowo et al, but in contrast to Raghunandan et al which showed the peak incidence at third and fourth decade of life. ${ }^{10,16,17}$ And Ramani et al which showed peak incidence at the age of 35-55 years. ${ }^{12}$

The most common type of psoriasis seen in our study was Psoriasis vulgaris otherwise known as plaque type of psoriasis $(82.22 \%)$ followed by erythrodermic psoriasis $(4.44 \%)$ and psoriatic arthritis. (4.44\%). It was similar to 
the findings in Raghunandan et al (90\%) and Ramani et al $(58 \%)$ which also showed plaque type of psoriasis was a most common type of psoriasis observed. ${ }^{12,15}$

The most common complaint of the presentation was itching in our study, followed by joint pain which was similar to the findings in Ayanlowo et al where common symptom was itching followed by pain and fever. ${ }^{10}$ Itching correlates with severity of psoriasis; leads to impairment in quality of life; the degree of stigmatization and depressive symptoms. ${ }^{18,19}$ Neurogenic inflammation with release of neuropeptides from dermal nerve endings; increased dermal vasculature and abnormal functioning of the opioid system among others were thought to be responsible for pruritus in psoriasis. ${ }^{19}$

In our study, distribution of the lesions was affecting the whole body in $34.4 \%$ followed by upper and lower limb in $32.22 \%$, scalp $28.88 \%$ and abdomen $23.33 \%$. This finding was in contrast to findings in a study by Raghunandan et al where lower limb was the most commonly affected body site $(90.9 \%)$, followed by upper limbs $(74.38 \%)$, head and neck $(62.80 \%)$ and trunk $(29.75 \%) .{ }^{16}$ Ramani et al, in $28.51 \%$ of cases the lower limb (single) was the most common initial site to be affected followed by generalized psoriasis (11.49\%), plantar surface $(11.49 \%)$ and other sites like the scalp, both lower limbs, trunk. ${ }^{12}$

Around 23 patients $(25.55 \%)$ in our study had associated comorbid disorder, most common being diabetes and hypertension this was similar to the findings in Bhandary et al and Raghunandan et al. ${ }^{11,16}$ According to the studies, there is a close association between metabolic syndrome and psoriasis. The prevalence of metabolic syndrome in the developed countries varies from 15 to $35 \% .^{20}$ One meta-analysis, it was concluded that, based on information, mostly from hospital-based studies, psoriasis is associated with cardiovascular disease. ${ }^{21}$

Most of the patients $(68 \%)$ were treated with the combination therapy in our study of systemic and topical drugs. With the average of 2.188 systemic drugs and 1.65 of topical drugs. Prevalence of polypharmacy in our study was $7 \%$. The most common group of systemic drugs used were Antihistaminic for the symptomatic treatment of itching. Another common group of the drug was multivitamins and antibiotics. Antibiotics were used to prevent the secondary infection as the majority of the patients had lesion involving the whole body. Among the drugs used specifically for psoriasis, Methotrexate was most commonly used both in the oral and parenteral route depending on the severity of the lesion. Similar findings were seen in Raghunandan et al, and Ramani et al other drugs used were cyclosporine, prednisolone, and apremilast, and one patient had received UVB therapy. ${ }^{12,16}$ The use of systemic anti-psoriatic drugs was less as most of the patients were managed with topical drugs, and only in severe cases, systemic drugs were prescribed.
The patients with comorbid disorder were also taking drugs for the disease concerned most common being the anti-diabetic medications like insulin, metformin and tenegliptin, and amlodipine as an antihypertensive drug. Hence it is essential also to take a detailed history regarding the comorbid disorder and the patient of the medication already been receiving to plan treatment in psoriatic patients.

There are various topical medications used in psoriatic patients with systemic drugs like Emollients (moisturizers), topical steroids, antifungals, and antibacterials. Among topical medication combination therapy of glucocorticoids with emollient like liquid paraffin or petroleum jelly and salicylic acid were used in most of the patients. The commonly used steroids were clobetasol propionate, halobetasol propionate, mometasone furoate, and betamethasone valerate. Similar to the findings were seen in the study by Ramani et al were salicylic acid; Halbetasole and clobetasol were commonly used. ${ }^{12}$ According to the study done by Raghunandan et al, glucocorticoid monotherapy is the most effective therapy. ${ }^{16}$ Antifungal drugs were used in our study in around $16 \%$ of patients for mainly in cases of scalp lesions of psoriasis along with the combination of salicylic acid only in $4.44 \%$ cases there was associated fungal lesion of the nail and other body parts.

Topical antibacterial drugs like fusidic acid and mupirocin were used to prevent secondary infection in patients with severe itching and extensive lesions. In only four patient's Vit D analog-like Calcipotriol was used. According to the study by Shwetha aryl, et al combination therapy with topical agents and NB UVB is beneficial in the treatment of psoriasis. ${ }^{1}$ However, phototherapy was not commonly used in our settings for the patients only one patient had received phototherapy.

As the present study was a retrospective study with a small sample size; hence, the generalization of the results to the population may not be appropriate. Because of the limited duration of the study with a cross-sectional design, the impact of pharmacotherapy on disease severity and progression could not be assessed.

\section{CONCLUSION}

We conclude that the plaque type of psoriasis is common in our settings with the fourth and fifth decade of the population getting affected. It can be managed and controlled using various treatment modalities that are available, including topical use of Emollients, Glucocorticoids, salicylic acid, Vit-D analogs like calcipotriol and systemic therapy with methotrexate and cyclosporine.

\section{ACKNOWLEDGEMENTS}

We want to thank medical superintendent for permitting and staff of medical records department, nursing staff in 
the dermatology ward for helping us to retrieve the patient's records.

Funding: No funding sources

Conflict of interest: None declared

Ethical approval: The study was approved by the Institutional Ethics Committee

\section{REFERENCES}

1. Aryal S, Xian L J, Zhi G S, Karki J. Comparative Study Of The Efficacy Of Various Topical Treatment Modalities And Phototherapy For Psoriasis Vulgaris: A Review. Int J Sci Inv Today. 2017;6(5):570-7.

2. Dogra S, Yadav S. Psoriasis in India: Prevalence and pattern. Indian $\mathrm{J}$ Dermatol Venereol Leprol. 2010;76:595-601

3. Jobling R. A patient's journey. Psoriasis. Br Med J. 2007;334:953-4.

4. Lebwohl M, Ali S. Treatment of psoriasis. Part1: Topical Therapy and Phototherapy. J Am Acad Dermatol. 2001;45:487-98.

5. Paolo Gisondi, Anna Ferrazzi, Giampiero Girolomoni. Metabolic comorbidities and Psoriasis. Acta Dermatovenerol Croat. 2010;18(4):297-304.

6. Di Meglio P; Villanova F; Nestle FO. Psoriasis. Cold Spring Harb Perspect Med. 2014;4:6.

7. Harden, J.L.; Krueger, J.G.; Bowcock, A.M. The immunogenetics of psoriasis: A comprehensive review. J Autoimmun. 2015;64:66-73.

8. Farber EM, Nall ML, Watson W. Natural history of psoriasis in 61 twin pairs. Arch Dermatol. 1974; 109,207-11.

9. Farber EM, Nall ML. The natural history of psoriasis in 5600 patients. Dermatologica. 1974;148:1-18.

10. Ayanlowo O, Akinkugbe A. Clinical pattern of psoriasis in patients seen at a tertiary hospital in Nigeria. J Clin Sci. 2016;13:137-42.

11. Bhandary PR, Sanath PK, Shetty NJ, Girish PN, Lathika K. Clinico- epidemiological study of Psoriasis and associated comorbidities. IAIM, 2016;3(3):118-22.
12. Ramani Y R, Panigrahy B, Mishra S, Singh BTPS.A study of treatment modalities in psoriasis in dermatology outpatient department of a tertiary care teaching hospital. Indian J Basic Applied Med Res. 2016;5(3):116-22.

13. Bennett PN, Brown MJ. Clinical Pharmacology. 10th Edition. USA: Churchill Livingstone; 2008: 274-290.

14. Pavithran K, Karunakaran M, Palit A, Raghunatha S. Disorders of keratinization. In: Valia RG, Valia AR, editors. IADVL Textbook of Dermatology. 3rd Edition, Volume 1. Mumbai: Bhalani Publishing House; 2000: 1021-1056.

15. Karamata VV, Gandhi AM, Patel PP, Sutaria A, Desai MK. A study of the use of drugs in patients suffering from psoriasis and their impact on quality of life. Indian J Pharmacol. 2017;49(1):84-8.

16. Raghunandan R, Pundarikaksha HP, Gopal MG. Pattern of drug use in the management of psoriasis in a tertiary care hospital: a prospective study. Int $\mathbf{J}$ Basic Clin Pharmacol. 2014;3:611-8.

17. Dogra S, Mahajan R. Psoriasis: Epidemiology, clinical features, co-morbidities, and clinical scoring. Indian Dermatol Online J. 2016;7:471-80.

18. Chang SE, Han SS, Jung HJ, Choi JH. Neuropeptides and their receptors in psoriatic skin in relation to pruritus. Br J Dermatol. 2007;156:1272-7.

19. Szepietowski JC, Reich A. Pruritus in psoriasis: An update. Eur J Pain. 2016;20:41-6.

20. Cameron AJ, Shaw JE, Zimmet PZ. The metabolic syndrome: Prevalence in worldwide populations. Endocrinol Metab Clin North Am. 2004;33:351-75.

21. Miller IM, Ellervik C, Yazdanyar S, Jemec GB. Meta - analysis of psoriasis, cardiovascular disease, and associated risk factors. J Am Acad Dermatol. 2013;69:1014-24.

Cite this article as: Gupta $S$, Acharya $S$, Shanmukha GB, Holla R. A pharmaco-epidemiological study of psoriasis. Int J Basic Clin Pharmacol 2019;8:2475-9. 